\title{
Lipoprotein (a) and Lipoprotein-associated Phospholipase A2 as Atherosclerosis Risk Factors (oxLDL) in Men with Central Obesity
}

\author{
Nelly Sari ${ }^{1,2^{*}}$, Andi Wijaya ${ }^{1,2}$, Ilhamjaya Patellongi ${ }^{2,3}$ \\ ${ }^{1}$ Prodia Clinical Laboratory, Jl. Cisangkuy No.2, Bandung, Indonesia \\ ${ }^{2}$ Post Graduate Program in Clinical Biochemistry, Hasanuddin University, Jl. Perintis Kemerdekaan Km 10, Makassar, Indonesia \\ ${ }^{3}$ Faculty of Medicine Hasanuddin University, Jl. Perintis Kemerdekaan Km 10, Makassar, Indonesia \\ *Correspondence: nelly.sari@prodia.co.id
}

\section{Abstract}

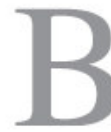

ACKGROUND: The increasing prevalence of obesity in Indonesia triggers a lot of research interest to overcome it. Obesity has a very important role as atherosclerosis and cardiovascular risk factors. The presence of oxidized LDL (oxLDL) on the vascular wall is a marker of atherosclerosis. The increase of Lipoprotein(a) (Lp(a)) and Lipoprotein associate phospholipase A2 (LpPLA2) occurs in patients with coronary artery disease (CAD), myocardial infarction, and unstable angina. It is well accepted that obesity is closely related to atherosclerosis and cardiovascular risk factors. However, correlation between Lp(a), LpPLA2 and oxLDL in central obesity has not yet been investigated. The aim of this study was to observing the correlation between $\mathrm{Lp}(\mathrm{a})$, LpPLA2 and oxLDL in early central obesity.

METHODS: An observational study with cross-sectional design on 76 men with central obesity, aged 30-67 years, was conducted. Central obesity was characterized by waist circumference $>90 \mathrm{~cm}$. Test of $L p(a)$ was performed by turbidimetric method and that of LpPLA2 was performed by sandwich enzyme immunoassay. Test of oxLDL was performed by ELISA. All statistical analyses were carried out using SPSS for Windows v.11.5 at a significance level of $\mathrm{p}<0.05$. The Pearson and Spearman's Rho correlation coefficient was used to assess the correlation between Lp(a), LpPLA2 and oxLDL. Obese men with acute inflammation (hsCRP $>10 \mathrm{mg} / \mathrm{L}$ ), renal failure
(Creatinine $>1.5 \mathrm{mg} / \mathrm{dL}$ ) and consumed antiinflammation were excluded from this study.

RESULTS: The concentration of LpPLA2 had a linear correlation $(r=-0.340, p=0.003)$ with the increase of oxLDL concentration. However, concentration of $\mathrm{Lp}(\mathrm{a})$ did not have linear correlation $(r=0.025)$ with increase of oxLDL concentration. This finding indicates that concentration of LpPLA2 had a negative correlation with increase of concentration of oxLDL. In addition, $\mathrm{Lp}(\mathrm{a})$ appears not to correlate with oxLDL significantly.

CONCLUSION: The study showed there was a significant correlation between concentration of LpPLA2 and concentration of oxLDL in men with central obesity. Higher concentration of LpPLA2 correlated with lower concentration of oxLDL.

KEYWORDS: Lp(a), LpPLA2, oxLDL, atherosclerosis, central obesity.

Indones Biomed J 2011; 3(1): 51-56

\section{Introduction}

The increasing prevalence of obesity in Indonesia has raised a lot of research interest in the pursuit of overcoming it. Central obesity has a very important role as a risk factor for atherosclerosis and cardivascular disease. Low Density 
Lipoprotein (LDL) is one precursor of atherosclerosis process. The presence of oxLDL on vascular wall can cause injury to the endothelial wall, thus triggering the entry of monocytes into the intima (1).

$\mathrm{Lp}(\mathrm{a})$ has a structure like LDL, which has apo(a) and single apolipoprotein B-100 (apo B-100) molecules that are linked by a disulfide bond. Increased concentration of $\mathrm{Lp}(\mathrm{a})$ is a risk factor for cardiovascular disease under the influence of prothrombotic and proatherogenic factors, including age, gender, race, and ethnicity background (2).

$\mathrm{Lp}$ (a) and oxidized phospholipids are hydrolysed by LpPLA2 (enzyme circulated in the blood bond to LDL cholesterol) to produce lysophosphatidylcholine which is proatherogenic. Some studies indicate that LpPLA2 can be used as an inflammatory marker of cardiovascular disease (3).

Several other studies have shown the increase of $\mathrm{Lp}$ (a) and LpPLA2 in subjects with CAD, myocardial infraction, and unstable angina. However, whether or not they have role as or correlation with atherosclerosis risk factors in early stage of central obesity has not yet been elucidated. The aim of this study was to observing the correlation between Lp(a), LpPLA2 and oxLDL in early central obesity.

\section{Methods}

\section{SUBJECTS}

The design of this study is an observational with cross sectional study, with minimum 68 samples. Eighty five men (aged 30-67 years) with central obesity were screened for the study. Central obesity was characterized by waist circumference $>90 \mathrm{~cm}$. However, 8 of the men were excluded from the study as they were found to have acute inflammation (hsCRP > $10 \mathrm{mg} / \mathrm{L}$ ), renal failure (creatinine $>1.5 \mathrm{mg} / \mathrm{dL}$ ), or consuming antiinflammatory drug. Therefore, only 76 subjects were fully involved in the study. The purpose of the study and the possible risks that might arise from it were informed to all subjects prior to informed consent signing. This study was approved by the review board of the Health Research Ethics Committee of the Faculty of Medicine, Hasanuddin University, Makassar, Indonesia. Before any assessment of biochemical markers was done,each of the study subjects was asked to complete a self administered questionnaires covering general data, medical history, usage of drug, smoking habit, activity, and alcohol intake.

\section{ASSAY OF BIOCHEMICAL MARKERS}

Height, body weight, waist circumference, and blood pressure were measured. All subjects were examined in the morning after overnight fasting for at least 10 hours. Creatinine and hsCRP were measured on all subjects to determine exclusion criteria. Measurement of creatinine was done by Jaffe RB \& C (Roche). Measurement of the level of hsCRP was done by solid phase chemiluminescent immunometric assay using Siemens manufactured reagent.

Lp(a) was measured by turbidimetric method using Daichii, Japan manufactured reagent. The PLAC ${ }^{\mathrm{TM}}$ Test Reagent Kit (diaDexus Inc. manufacturer, South San Fransisco, United Stated), a turbidimetric immunoassay using two highly specific monoclonal antibodies for direct measurement of LpPLA2 concentration, was used. OxLDL in EDTA plasma /serum/plasma heparin (oxLDL) was measured by sandwich / capture ELISA based on the mouse monoclonal antibody 4E6 (Mercodia manufacturer, Sweden).

\section{STATISTICAL ANALYSIS}

All statistical analysis were performed with SPSS windows ver 11.5 statistical software package. General descriptive data analysis, univariate analysis methods were used to calculate mean, maximum - minimum value and standard deviation. Normality analysis data using non-parametric test Kormogorov Smirnov. We also used Pearson or Spearman correlation statistic to examine the relationship between variables.

\section{Results}

General characteristics of the study subjects are summarized in Table 1. In general, the population characteristics of the study subjects were in compliance with the inclusion and exclusion criteria.

The correlation between $\mathrm{Lp}$ (a) and oxLDL is shown in Figure 1, as results of analysis using bivariate correlation test of Pearson and represented in the form of a scatter graph.

To determine the correlation between LpPLA2 and oxLDL, the data were analyzed by using Pearson bivariate analysis (Figure 2). To assess the correlation between Lp(a) and LpPLA2, we used Pearson bivariate analysis test. The results are presented in a scatter graph, as shown in Figure 3. 
Table 1. General Characteristics of Subjects

\begin{tabular}{lccccc}
\hline Variable & N & Minimum & Maximum & Mean & Std. Deviation \\
\hline Age (years) & 76 & 30.00 & 67.00 & 46.18 & 9.93 \\
Height $(\mathrm{cm})$ & 76 & 156.00 & 186.00 & 168.21 & 6.46 \\
Weight $(\mathrm{kg})$ & 76 & 64.00 & 120.00 & 81.01 & 12.28 \\
BMl $\left(\mathrm{kg} / \mathrm{m}^{2}\right)$ & 71.72 & 41.91 & 28.61 & 3.88 \\
Waist circumference $(\mathrm{cm})$ & 76 & 90.00 & 125.00 & 100.62 & 8.06 \\
Lp(a) (mg/dL) & 76 & 1.00 & 68.00 & 10.00 & 11.28 \\
LpPLA2 (mg/dL) & 76 & 109.17 & 419.58 & 233.82 & 70.88 \\
OxLDL (mU/L) & 76 & 83097.67 & 205736.66 & 149626.74 & 33676.48 \\
Valid N & 76 & & & & \\
\hline
\end{tabular}

BMI = Body Mass Index; Lp(a) = Lipoprotein (a); LpPLA2 = Lipoprotein-Associated Phospholipase A2; oxLDL = Oxidized Low-Density Lipoprotein.

Table 2. Non Parametric Test (Kormogorov-Smirnov)

\begin{tabular}{lcc}
\hline & Kormogorov-Smirnov & $\mathbf{P}$ \\
\hline Waist circumference & 0.937 & 0.344 \\
Lp(a) & 1.990 & 0.001 \\
LpPLA2 & 0.452 & 0.987 \\
OxLDL (mU/L). & 1.189 & 0.118 \\
\hline
\end{tabular}

Lp(a) = Lipoprotein (a); LpPLA2 = Lipoprotein-Associated Phospholipase A2; oxLDL = Oxidized Low-Density Lipoprotein. 


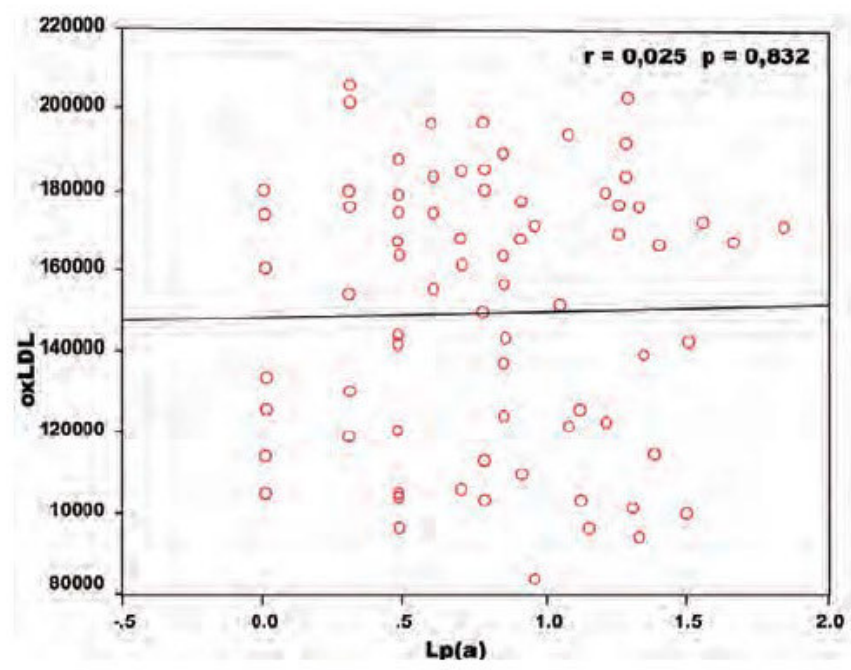

Figure 1. Correlations between Lp(a) and oxLDL.

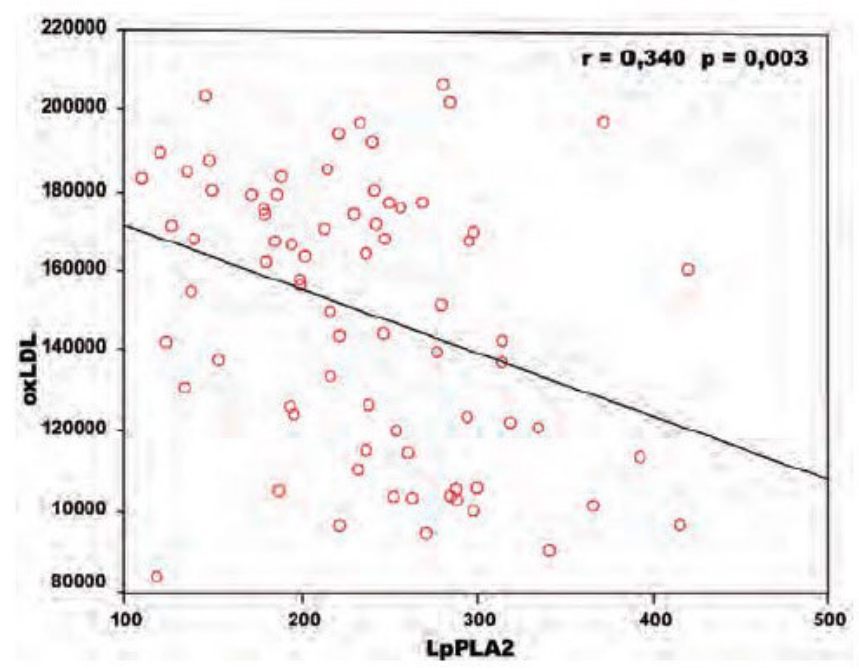

Figure 2. Correlations between LpPLA and oxLDL.

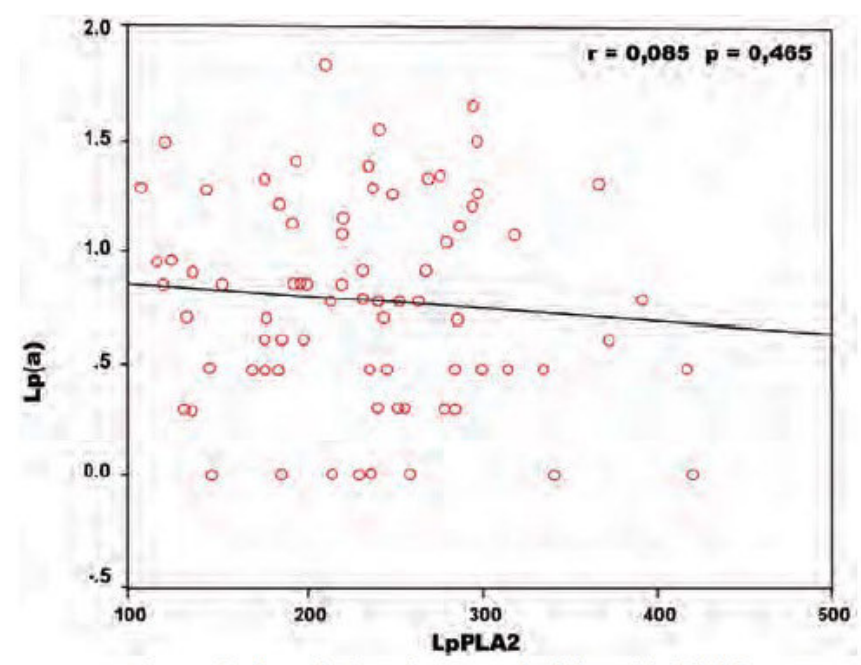

Figure 3. Correlations between $\mathrm{Lp}(\mathrm{a})$ and LpPLA2. 


\section{Discussion}

In this study, we only studied male subjects with central obesity on the consideration that in the general population men have higher prevalence of central obesity than women. In addition to this fact, much evidence has confirmed that the biomarker $\mathrm{Lp}(\mathrm{a})$ and LpPLA2 are affected by gender. $(4,5,6)$

By using bivariate Pearson correlation test between $\mathrm{Lp}$ (a) and oxLDL, we found there was no linear correlation between the two parameters $(r=0.025, p=$ 0.832 ). This might be due to fact that $L p(a)$ is indirectly affected by the level of oxLDL and very much directly affected by gender (2). Another explanation is that there is a specific transportation system which in turn can increase accumulation of cholesterol in cells (7). OxLDL and Lp(a) will be accumulated in a large amount in the macrophages to form fatty streak and foam cells. In the initiation process of aterosclerosis, $\mathrm{Lp}(\mathrm{a})$ is involved in triggering adhesion molecules (8).

The study by Pillariseti et al. in1997 has found that high plasma concentration of $\mathrm{Lp}$ (a) caused a stronger atherogenic characteristic as compared to natural LDL; this was due to the role of $L p(a)$ in increasing affinity to arterial intimal proteoglycans that further increased concentration of LDL and oxLDL (9). In our study, the mean concentration of $\mathrm{Lp}$ (a) was $10 \mathrm{mg} / \mathrm{dL}$, indicating a weak affinity of $\mathrm{Lp}(\mathrm{a})$ and oxLDL and another explanation on why there was no linear correlation between the two parameters.

Although the concentration of $\operatorname{Lp}(a)$ does not have a linear correlation with that of oxLDL in central obese men, the increase of $\mathrm{Lp}(\mathrm{a})$ is parallel to the increase of OxLDL. This result supports result of previous studies that confirmed increase of $\mathrm{Lp}$ (a) was a risk factor for cardiovascular disease and stroke $(2,5)$.

The similarity in the structure of $\mathrm{Lp}$ (a) and LDL, in which $\mathrm{Lp}(\mathrm{a})$ is a large molecule and has Apo(a) and apoB-100 attached by disulfide bond, hence both $\mathrm{Lp}$ (a) and LDL are very sensitive to oxidative process, increases the ability of forming foam cells and involvement in early stage of atherosclerosis $(10,5)$. An increased level of $\mathrm{Lp}$ (a) induces monocyte circulation (11) and increases expression of intercellular adhesion of molecules (12).

In this study the concentration of $\mathrm{Lp}(\mathrm{a}) \mathrm{had}$ a wide range, with a minimum of $1.0 \mathrm{mg} / \mathrm{dL}$ and a maximum of $68.0 \mathrm{mg} / \mathrm{dL}$. This wide variation was due to the fact that $90 \%$ of the concentration of $\mathrm{Lp}(\mathrm{a})$ is controlled by gen locus which codes apo(a) protein. Apo(a) polymorphism is affected by more than 34 alleles. Moreover, concentration of $L p(a)$ is affected by ethnicity. Concentration of $L p(a)$ varies among individuals (ranges from $<1 \mathrm{mg} / \mathrm{dL}$ up to $>1 \mathrm{~g} / \mathrm{L}$ ) and among populations, the highest is among the black race (13).

Bivariate Pearson correlation test showed there was a correlation between LpPLA2 and oxLDL $(r=-0.340$; $\mathrm{p}=0.003$ ). This finding supports the previous studies that showed LpPLA2 was related to stroke occurrence (14). Other study of NOMAS (Nothern Manhattan Stroke Study) found LpPLA2 correlated with the increased incidence of stroke, heart attack, and other death causing vascular diseases.

Negative $r$ value reflected a significance decline of LpPLA2 ( $p=0.003$ ) which added the rise of oxLDL. This finding supports the theory stating that in human LpPLA2 is in circulation and $80 \%$ binds to LDL. Oxidative process on phospholipid (oxPL) or on LDL (oxLDL) is a substrate for LpPLA2 enzyme (15). LpPLA2 hydrolises oxidized phospholipid in oxLDL that results in lysophosphatidylcholine and oxidized fatty acid, which is a proinflammatory (atherogenic) substance and act to accelerate atherosclerosis process by stimulating endothelial cells to produce proinflammatory cytokines $(3,16)$. In this study we measured the mass of LpPLA2 instead of its activity, which does not make any difference since the mass of LpPLA2 as well as its activity can be used as an independent predictor of the atherosclerosis process (17).

In this study we found out there was no linear correlation between Lp(a) and LpPLA2 $(r=-0.085 ; \mathrm{p}=$ 0.465). The distribution of LpPLA2 on HDL and LDL is affected by the presence of $L p(a)$ when the plasma level of lipoprotein(a) is higher than $30 \mathrm{mg} / \mathrm{dL}$ (18). However in this study the mean plasma level of $\mathrm{Lp}$ (a) was $10 \mathrm{mg} / \mathrm{dL}$, thus there was no significant correlation found between Lp(a) and LpPLA2. Other studies have shown they would be a bond of $L p(a)$ and LpPLA2 when it had mass 1.5-2 times greater than that of the enzyme and its activity was higher than that of LpPLA2 $(18,19)$.

The negative $r$ value showed increase of $L p(a)$ was affected by the decrease of LpPLA2, though the correlation had shown no significance. The reason for it might be that particle of $\mathrm{Lp}$ (a) is easily oxidized due to its LDL-like structure (10), and proinflammatory oxidized phospholipid is easily binded to $\mathrm{Lp}(\mathrm{a})$, and in turn will be taken up by the macrophage to form foam cells. Lipoprotein (a) is a substrate for LpPLA2 modification, thus the decline of $\mathrm{Lp}(\mathrm{a})$ is parallel with the increase of LpPLA2 (11). The same conclusion was made by other studies by Tsironis $e t$ $a l$. in 2004 which found patients with CAD had mass and activity of LpPLA2 lower than that of Lp(a). 


\section{Conclusion}

This study showed that there is no linear relationship between elevated concentration of Lp (a) and elevated concentration of oxLDL in men with central obesity, but there is a linear relationship $(r=-0.340 \mathrm{p}=0.003)$ between decreased concentrations of oxLDL and elevated concentration of LpPLA2. The greater the concentration of oxLDL, the lower the concentration of LpPLA2.

\section{Acknowledgements:}

We thank the Prodia Education and Research Institute for the invaluable support given to this study.

\section{References:}

1. Koenig W, Khuseyinov N. Biomarkers of Atherosclerotic Plaque Instability and Rupture. Arterioscler Thromb Vasc Biol. 2007; 27: 15-26.

2. Scanu AM. Lp(a) lipoprotein - Coping With Heterogeneity. N Engl J Med. 2003; 349: 2089-90.

3. MacPhee CH, Moores KE, Boyd HF, Dhanak D, Ife RJ, Leach CA, et.al. Lipoprotein-associated phospholipase A2, Platelet-Activating Factor Acetylhydrolase, Generates Two Bioactive Products During The Oxidation of Low-Density Lipoprotein: Use of A Novel Inhibitor. Biochem J. 1999; 338: 479-87.

4. McCormick PAS. Lipoprotein (a): Biology and Clinical Importance. Clinical Biochemistry. 2004; 25: 69-80.

5. Ariyo AA, Chau Thach, and Tracy R. Lp(a) Lipoprotein, Vascular Disease, and Mortality in the Elderly. N Eng J Med. 2003; 349: 2108-15.

6. Iribarren C, Gross MD, Darbinian JA, Jacobs, Jr D.R., Sidney, S., Loria, C. Relation of Lipoprotein-associated phospholipase A2 Mass and Activity with Coronary Artery Calcification in Young Adults: the CARDIA Study. Arterioscler Thromb Vasc Biol. 2005; 25:1.
7. Bottalico LA, Keesla GA, Fless GM and Tobas I, J Biol Chem., 1993; 268: 8569-73.

8. Allen S, Khan S, Tam Sp, Koschinsky M, Taylor P, Yacoub M. Expression of Adhesion Molecules by $L p(a)$ : A Potential Novel Mechanism for its Atherogenicity. FASEB J.,1998. $12,1765-76$.

9. Bergmark C, Dewan A, Orsoni A, Merki S, Miller ER, Min-Jeong Shin, et.al. Anovel function of lipoprotein (a) as a preferential carrier of oxidized phospholipids in human plasma. J Lipid Research. 2008; 49: 2230-9.

10. Danesh J, Collins R, and Peto R. Lipoprotein(a) and Coronary Heart Disease Meta-Analysis of Prospective Studies. Circulation; 2000: 1082-5.

11. Syrovets T, Thillet J., Chapman MJ, Simmet T. Lipoprotein (a) is Potent Chemoattractant for Human Peripheral Monocytes. Blood. 1997; 90: 2027-36.

12. Takami S, Yamashita S, Kihara S, Ishigami M, Takemura K, Kume N, et.al. Lipoprotein (a) enhances the expression of intercellular adhesion molecule-1 in cultured human umbilical vein endothelial cells. Circulation. 1998; 97: 721-8.

13. Cano EA. Structural Basis for the Pathophysiology of Lipoprotein (a) in The Athero-trombotic Process. Brazilian J. Med. Bio. Res. 1997; 30: 1271-80.

14. Oei HH, van der Meer IM, Hofman A, Koudstaal PJ, Stijnen, T, Breteler MM, et.al. Lipoprotein associated phospholipase A2 activity is associated with risk of coronary heart disease and ischemi stroke: The Rotterdam study. Circulation. 2005; 111: 570-5.

15. Lerman A \& McConnel JP. Lipoprotein-associated phospholipase A2: a risk marker or a risk factor?. Am J Cardiol. 2008; 101: $11 \mathrm{~F}-22 \mathrm{~F}$.

16. Sudhir K. Clinical review: lipoprotein-associated phospholipase A2, a novel inflammatory biomarker and independent risk predictor for cardiovascular disease. J. Clin Endocrinol Metab. 2005; 90: 3100-5.

17. Persson M, Nilsson J, Nelson JJ, Hedblad B, Berglund G. The epidemiology of LpPLA2: Distribution and correlation with cardiovascular risk factors in a population-based cohort. Atherosclerosis 2007; 190: 388-96

18. Karabina SAP, Elisaf MC, Goudevenos J, Siamopoulos KC, Sideris D, Tselepis AD. PAF-Acetylhidrolase activity of $L p(a)$ before and during $\mathrm{Ce}(2+)$-induced oxidatived modification in vitro. Atherosclerosis 1996; 125: 121-34.

19. Tsironis ID, Katsouras CS, Lourida E, Mitsios JV, Goudevenos $\mathrm{J}$, Elisaf $\mathrm{M}$, et al. Reduced PAF-acetyllhydrolase activity associate with $L p(a)$ patients with coronary artery disease. Atherosclerosis 2004; 177: 193-201. 